\title{
Application of bronchoscopic argon plasma coagulation in the treatment of tumorous endobronchial tuberculosis: Historical controlled trial
}

\author{
Faguang Jin, MD, PhD, ${ }^{a}$ Deguang Mu, MD, PhD, ${ }^{\mathrm{b}}$ Yonghong Xie, MD, PhD, ${ }^{\mathrm{b}}$ Enqing Fu, MD, ${ }^{\mathrm{b}}$ \\ and Youmin Guo, MD, $\mathrm{PhD}^{\mathrm{a}}$
}

Objective: The purpose of this study was to evaluate the efficacy and safety of bronchoscopic argon plasma coagulation for tumorous endobronchial tuberculosis.

\begin{abstract}
Methods: We analyzed the records of 115 patients with tumorous endobronchial tuberculosis who did not show luminal narrowing of the bronchus at diagnosis. Of these 115 patients, 41 patients received bronchoscopic argon plasma coagulation plus routine antituberculosis chemotherapy (argon plasma coagulation group) and the other 74 patients received only routine antituberculosis chemotherapy (chemotherapy group). The treatment effects between these 2 groups were compared based on changes in lesions, rate of lesion disappearance, and complications associated with bronchoscopic argon plasma coagulation.
\end{abstract}

Results: The complete removal rate was $100 \%$ in patients in argon plasma coagulation group. About $84.6 \%$ lesions disappeared completely in patients in the chemotherapy group. The rate of disappearance of lesions in the argon plasma coagulation group was faster than that of the chemotherapy group. There were no severe complications in the argon plasma coagulation group.

Conclusions: Bronchoscopic argon plasma coagulation can accelerate the healing of tumorous endobronchial tuberculosis and can help prevent progressive bronchial stenosis resulting from tumorous endobronchial tuberculosis, and it is a very safe method. (J Thorac Cardiovasc Surg 2013;145:1650-3)

Endobronchial tuberculosis (EBTB) is defined as a tuberculous infection of the tracheobronchial tree with microbial and histopathologic evidence. EBTB was divided mainly into 7 types according bronchoscopic features: actively caseating, edematous-hyperemic, fibrostenotic, tumorous, granular, ulcerative, and nonspecific bronchitic. And a certain proportion of tumorous EBTB changed into the fibrostenotic type. $^{1,2}$

The most important sequela of EBTB is bronchial stenosis, which causes wheezing, dyspnea, and obstructive pneumonia. Various bronchoscopic techniques applied to treat stenotic lesions include laser and cryosurgery through a fiber-optic bronchoscope. ${ }^{3-5}$ Granted bronchoscopic treatment may have some efficacy, there still have been no reports of the use of argon plasma coagulation (APC) in patients with EBTB, but without luminal narrowing at the time of diagnosis. Whether this technique can help

From the Center of Molecular Imaging, ${ }^{\text {a }}$ First Affiliated Hospital of Medical School, Xi' an Jiaotong University, Xi' an, Shaanxi Province, China; and the Department of Respiratory Disease, ${ }^{\mathrm{b}}$ Tangdu Hospital, Fourth Military Medical University, Xi' an, Shaanxi Province, China.

Disclosures: Authors have nothing to disclose with regard to commercial support. F.J., D.M., and Y.X. are co-first authors.

Received for publication May 23, 2012; revisions received Aug 2, 2012; accepted for publication Aug 16, 2012; available ahead of print Sept 21, 2012.

Address for reprints: Youmin Guo, MD, PhD, Center of Molecular Imaging, First Affiliated Hospital of Medical School, Xi' an Jiaotong University, No. 277 Yantaxilu Rd, Xi'an, Shaanxi Province, P.R. China 710061 (E-mail: jinfag@ @otmail.com). $0022-5223 / \$ 36.00$

Copyright (c) 2013 by The American Association for Thoracic Surgery http://dx.doi.org/10.1016/j.jtcvs.2012.08.042 prevent tumorous EBTB from progressing into stenosis needs to be clarified.

In this study, we analyzed the records from our hospital of 115 patients with tumorous EBTB to assess the efficacy and safety of bronchoscopic APC treatment.

\section{METHODS}

\section{Study Subjects}

Before June 2007, we applied routine antituberculosis chemotherapy alone for tumorous EBTB. Several cases developed into bronchial stenosis during the course of treatment. We applied APC in these cases and they got completely cured at last. Then, after June 2007, we began to apply APC for tumorous EBTB patients with no bronchial stenosis. We analyzed the records of 241 patients who were diagnosed as and treated for tumorous EBTB in our department from February 2004 through January 2009.

The study was approved by the medical ethics committee of the Fourth Military Medical University. The two diagnostic criteria of tumorous EBTB and categories for patient selection were as follows:

1. Diagnosis of tuberculosis was confirmed by microbiologic or histopathologic examination. All patients underwent bacteriologic studies of sputum and bronchoscopic aspirates, and they also underwent pathologic studies of bronchoscopic biopsy specimens.

2. The tumorous EBTB was defined according to the work of Chung and Lee, ${ }^{1}$ as shown in Figure 1.

We selected 115 patients in our study. Inclusion criteria were as follows: (1) the sputum smears were positive for tubercle bacillus, (2) the sputum culture showed no multidrug-resistant tuberculosis (MDR-TB), and (3) the treatment and follow-up were completed. The exclusion criteria were as follows: (1) patients had an antituberculous medication history when they presented to us, (2) patients had other subtypes of EBTB or it had already developed into bronchial stenosis, (3) the sputum culture showed 


\section{Abbreviations and Acronyms}

APC $=$ argon plasma coagulation

EBTB = endobronchial tuberculosis

MDR-TB = multidrug-resistant tuberculosis

multidrug resistance, and (4) patients who did not complete our treatment or follow-up.

In this study, multidrug resistance was referred to as any combination of resistance to rifampicin, isoniazid, or ethambutol.

\section{Treatment Procedure}

Endobronchial APC was performed with an APC unit (ARCO 3000; Soring Co, Quickborn, Germany) via an electronic bronchoscope (model BF-260; Olympus Co, Tokyo, Japan). Energy at 30 to $40 \mathrm{~W}$ and argon flow at 0.3 to $2.0 \mathrm{~L} / \mathrm{min}$ was applied through a $2.0-\mathrm{mm}$ diameter, 150-cm-long APC monopolar probe. The probe was inserted through the working channel of the bronchoscope. The APC probe was positioned 1 to $2 \mathrm{~mm}$ away from the mucosa, and 1- to 2-second pulses of ablation were repeated until the lesion was coagulated. The devitalized tissue was removed mechanically with grasping forceps. Bronchoscopic APC was performed every 2 weeks and was terminated when there was no tumorous bronchial tuberculosis lesions observed.

Routine antituberculosis chemotherapy was the conventional combination of isoniazid, rifampicin, ethambutol, and pyrazinamide for 2 months followed by isoniazid and rifampicin for another 4 months.

\section{Bronchoscopic Examination and Follow-up}

These patients received bronchoscopic examination every 2 weeks from the beginning of the treatment for 6 months. Then, 6 months later, the patients received a bronchoscopic examination once every month for 3 months.

\section{Outcome Events}

The outcome measures were the lesion changes, the lesion disappearance rate, and the complications of bronchoscopic APC. The changes in lesion were categorized into 4 groups: (1) improved, the number and/or the volume of lesions was reduced; (2) healed, the lesions were removed completely; (3) stationary, there were no changes in the number and/or the volume of the lesions; and (4) worsened, the volume and/or the number of lesions increased.

\section{RESULTS}

Of the 115 patients, 41 received bronchoscopic APC plus routine antituberculosis chemotherapy and 74 received only the routine antituberculosis chemotherapy. Table 1 shows the characteristics of the study population. The main symptoms were fever, irritating cough, and chest pain. Neither dyspnea nor obstructive pneumonia was seen at patient presentation. Figure 1 shows the features of tumorous EBTB, treatment of bronchoscopic APC, and the treatment result of the lesion.

\section{Localization of Lesions}

There were 180 lesions found in these 115 patients. The localization and number of lesions were as follows: trachea, 7 (3.9\%); left main bronchus, 52 (28.9\%); left upper lobar bronchus, $28(15.6 \%)$; left lingual lobar bronchus, $13(7.2 \%)$; left lower lobar bronchus, 20 (11.1\%); right main bronchus, 17 (9.4\%); right upper lobar bronchus, 15 (8.3\%); right middle lobar bronchus, 15 (8.3\%); right lower lobar bronchus, $13(7.2 \%)$.

\section{Change in Lesions}

Of those 74 patients in the conventional chemotherapy group, 62 patients were healed completely, with a treatment duration of 5 months. The lesions in the remaining 12 patients showed no improvement after 4 months of treatment. To remove the lesions in these 12 patients, we applied APC and the lesions healed completely (Table 2).

Of those patients in the APC group, the mean APC treatment was about 3 , and all these patients healed completely. The duration from the start of therapy to the complete disappearance of lesions was about 1.5 months. No stenosis appeared afterward.

\section{Follow-up Result}

Six months later, the patients received bronchoscopic examination once every month for 3 months. No lesion recurrence was observed.

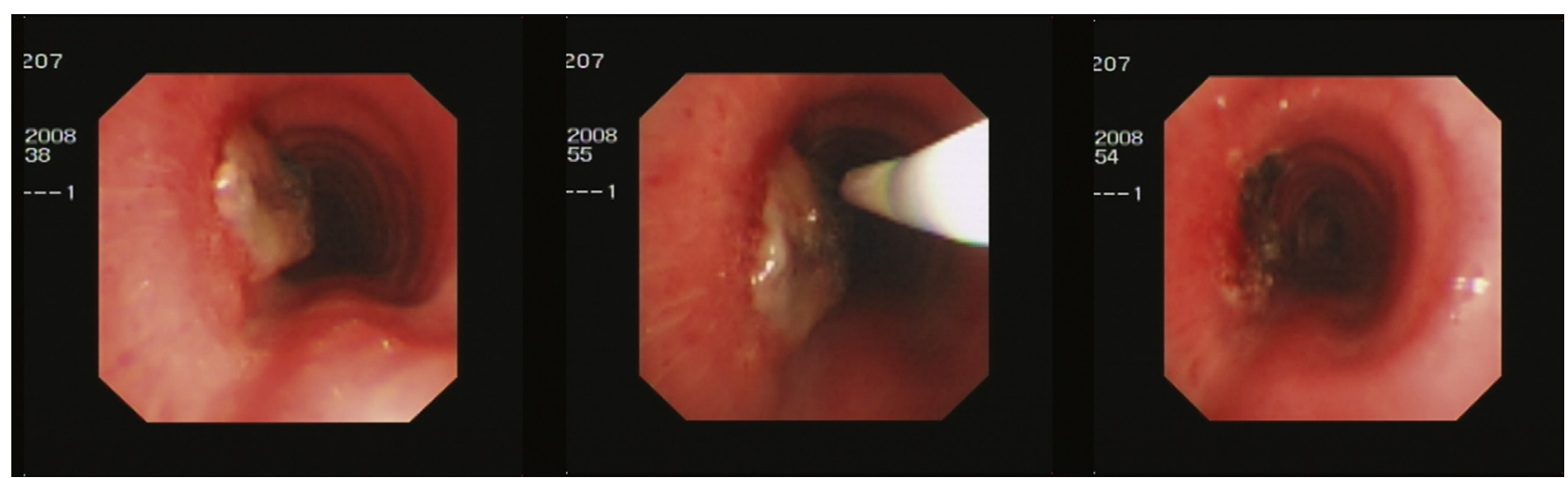

FIGURE 1. Bronchoscopic featuring and treatment result of tumorous endobronchial tuberculosis. Left, Bronchoscopic feature of tumorous endobronchial tuberculosis. Middle, Treating the lesion. Right, Treatment result. 
TABLE 1. Clinical characteristics of 115 patients

\begin{tabular}{lcc}
\hline \multicolumn{1}{c}{ Characteristic } & $\begin{array}{c}\text { Bronchoscopic } \\
\text { APC }+ \\
\text { chemotherapy }\end{array}$ & $\begin{array}{c}\text { Antituberculosis } \\
\text { chemotherapy } \\
\text { alone }\end{array}$ \\
\hline Sex & & \\
Female, $\mathrm{n}(\%)$ & $30(73.1 \%)$ & $48(64.9 \%)$ \\
Age, y, mean \pm SD & $30.2 \pm 10.4$ & $33.1 \pm 11.3$ \\
Location of airway lesions, & & \\
$\quad \mathrm{n}(\%)$ & $2(3.5 \%)$ & $5(4.1 \%)$ \\
Trachea & $5(8.8 \%)$ & $12(9.8 \%)$ \\
Right main bronchus & $7(12.3 \%)$ & $8(6.5 \%)$ \\
Right upper lobar bronchus & $5(8.8 \%)$ & $10(8.1 \%)$ \\
Right middle lobar bronchus & $3(5.3 \%)$ & $10(8.1 \%)$ \\
Right lower lobar bronchus & $19(33.3 \%)$ & $33(26.8 \%)$ \\
Left main bronchus & $8(14.0 \%)$ & $20(16.3 \%)$ \\
Left upper lobar bronchus & $2(3.5 \%)$ & $11(8.9 \%)$ \\
Left lingual lobar bronchus & $6(10.5 \%)$ & $14(11.4 \%)$ \\
Left lower bronchus & & \\
\hline APC, Argon plasma coagulation. & &
\end{tabular}

\section{Complications}

During our APC treatment, complications included laryngeal spasm $(1 / 41,2.4 \%)$, cough $(35 / 41,85.4 \%)$, and a small amount of bleeding $(5-10 \mathrm{~mL} ; 5 / 41,12.2 \%)$. No secondary pulmonary infection, esphagotrachea fistula, pneumothorax, trachea perforation, or death occurred.

\section{DISCUSSION}

Endobronchial tuberculosis has been reported in $10 \%$ to $37 \%$ of patients with pulmonary tuberculosis, and $>90 \%$ of the patients with tracheobronchial tuberculosis have some degree of bronchial stenosis. ${ }^{6-8}$ In recent years, the incidence of pulmonary tuberculosis shows an increasing tendency in China, ${ }^{9}$ which results in an increasing incidence of tracheobronchial tuberculosis. Despite adequate antituberculosis chemotherapy, the tracheobronchial tuberculous lesions in many patients heal by fibrostenosis, ${ }^{10,11}$ which results in bronchial obstruction and various complications such as atelectasis and obstructive pneumonia. Thus, it is important to prevent bronchial stenosis and complications in patients with EBTB.

With the development of interventional bronchoscopic techniques, many new methods such as laser, APC, and stent are used to treat bronchial stenosis. ${ }^{12}$ Until now, the literature reported that these treatment methods were not applied until the stenosis occurred.
In APC, a high-frequency electrical current is transferred from the probe tip through ionized argon gas, without contact with the tissue, resulting in superficial thermal coagulation. The procedure is very effective for hemostasis, and the visible ionized argon plasma can be guided visually. The penetration depth of about $2 \mathrm{~mm}$ is broadly constant and predictable, and limits thermal trauma, thereby avoiding unnecessary damage to the airway cartilage and connective tissue. APC also has advantages such as mobility and a lower cost than other commonly used modalities.

Whether APC can help prevent tumorous EBTB from progressing to stenosis needs to be clarified. To this end, we analyzed the treatment records of 115 patients with tumorous EBTB in our hospital to determine the efficacy and safety of bronchoscopic APC treatment. In our study, of the 41 patients in the APC plus antituberculosis group, all patients healed completely. After 4 weeks, about $36.6 \%$ of the patients in the APC + chemotherapy group were healed whereas about $10.8 \%$ of chemotherapy-alone group were healed. At 16 weeks, all patients in the APC + chemotherapy group were healed completely whereas about $67.6 \%$ of patients in the chemotherapy-alone group were healed. Therefore, we believe that the APC treatment can prevent bronchial stenosis caused by tumorous EBTB and can accelerate the rate of healing in contrast with antituberculosis chemotherapy alone.

Granted, our study shows that APC accelerates the rate of healing of tumorous EBTB, but additional study could include investigations into the efficacy of a shorter systemic treatment to avoid toxicity, although we do not believe systemic treatment can be administered for a shorter period of time. In our experience, some patients terminated systemic treatment themselves. Unfortunately, the lesions in all these patients recurred.

The cases had not showed bronchial stenosis, under this circumstance, lung function might not be a sensitive measurement to judge the patients' condition, so we did not perform the lung function test for the patients. Whether lung function was impaired by this kind tumorous EBTB or improved by our treatment needs to be studied further.

The complications caused by the use of bronchoscopy have a trend to increase during these years, ${ }^{13}$ but in our study no severe complications occurred during the APC treatments. During our treatment, the main complications

TABLE 2. Bronchoscopic findings of these 2 groups of patients

\begin{tabular}{|c|c|c|c|c|c|c|c|c|}
\hline \multirow[b]{2}{*}{ Time point (wk) } & \multicolumn{4}{|c|}{ APC + chemotherapy, $n$} & \multicolumn{4}{|c|}{ Chemotherapy alone, $n$} \\
\hline & Healed & Improved & No change & Worse & Healed & Improved & No change & Worse \\
\hline 4 & 15 & 26 & 0 & 0 & 8 & 52 & 11 & 3 \\
\hline 8 & 41 & 0 & 0 & 0 & 21 & 41 & 9 & 3 \\
\hline 12 & 41 & 0 & 0 & 0 & 33 & 29 & 9 & 3 \\
\hline 16 & 41 & 0 & 0 & 0 & 50 & 12 & 9 & 3 \\
\hline
\end{tabular}

$A P C$, Argon plasma coagulation. 
were laryngeal spasm, cough, and a small amount of bleeding. The main reason for laryngospasm was because of insufficient anesthesia and violent insertion of the bronchoscope, which stimulated the vocal cords. This complication also occurred during other bronchoscopic procedures and we reasoned it had no relationship with APC. The cough observed during APC was stimulated by the gasified tissues but did not make these patients suffer much. The bleeding could be stopped by the APC treatment itself. Although APC caused no severe complications, the bronchoscopic operation itself had some risks. If the patients had severe underlying diseases such as heart failure or a bleeding tendency, we would not use APC. Although our results are promising, in that we observed patients for 9 months, it is our opinion that long-term follow-up should be done and add additional support to our findings. From our study we deem that APC could be considered an effective and safe method for management of tumorous EBTB.

\section{References}

1. Chung HS, Lee JH. Bronchoscopic assessment of the evolution of endobronchial tuberculosis. Chest. 2000;117:385-92.
2. Kim YH, Kim HT, Lee KS, Uh ST, Cung YT, Park CS. Serial fiberoptic bronchoscopic observations of endobronchial tuberculosis before and early after antituberculosis chemotherapy. Chest. 1993;103:673-7.

3. Dumon JF, Reboud E, Garbe L, Aucomte F, Meric B. Treatment of tracheobronchial lesions by laser photoresection. Chest. 1982;81:278-84.

4. Jeong BH, Um SW, Suh GY, Chung MP, Kwon OJ, Kim H, et al. Results of interventional bronchoscopy in the management of postoperative tracheobronchial stenosis. J Thorac Cardiovasc Surg. 2012 (Epub ahead of print).

5. Marasso A, Gallo E, Massaglia GM, Onoscuri M, Bernardi V. Cryosurgery in bronchoscopic treatment of tracheobronchial stenosis: indications, limits, personal experience. Chest. 1993;103:472-4.

6. Han JK, Im JG, Park JH, Han MC, Kim YW, Shim YS. Bronchial stenosis due to endobronchial tuberculosis: successful treatment with self-expanding metallic stent. AJR Am J Roentgenol. 1992;159:971-2.

7. So SY, Lam WK, Yu DY. Rapid diagnosis of suspected pulmonary tuberculosis by fiberoptic bronchoscopy. Tubercle. 1982;63:195-200.

8. Judd AR. Tuberculous tracheobronchitis: a study of 500 consecutive cases. $J$ Thorac Surg. 1947;16:512-23.

9. Zhu LZ. The treatment of MDR-TB. Chin J Tuberc Respir Dis. 2000;23:77-8 (in Chinese).

10. Smith LS, Schillaci RF, Sarlin RF. Endobronchial tuberculosis: serial fiberoptic bronchoscopy and natural history. Chest. 1987;91:644-7.

11. Hoheisel G, Chan BK, Chan CH, Chan KS, Teschler H, Costabel U. Endobronchial tuberculosis: diagnostic features and therapeutic outcome. Respir Med 1994;88:593-7.

12. Low SY, Hsu A, Eng P. Interventional bronchoscopy for tuberculosis tracheobronchial stenosis. Eur Respir J. 2004;24:345-7.

13. Jin F, Mu D, Chu D, Fu E, Xie Y, Liu T. Severe complications of bronchoscopy. Respiration. 2008;76:429-33. 